\title{
El Club de Inglés y las destrezas comunicativas Caso: evaluadores de emergencia ECU 911 -Portoviejo
}

The English Club and the communicative skills. Case: Emergency evaluators ECU-91 1 Portoviejo

\author{
LETICIA CEDEÑO* (D), ROSALYN CEDEÑO, (D \\ ROSARIO FLORES (D), JOEL LOOR (D) \\ Universidad Técnica de Manabí, \\ Av. Urbina y Portoviejo 130105, Portoviejo - Ecuador \\ (*) leticia.cedeno@utm.edu.ec
}

\section{RESUMEN}

Manabí se está convirtiendo en una provincia turística, visitada por miles de personas de todo el mundo durante diferentes épocas del año, pues sus múltiples atractivos se dan a conocer a través de las redes sociales y de las agencias de viaje que invitan a los extranjeros a visitar las hermosas playas, ríos, badenes, parques y museos de esta ciudad, así como a degustar la exquisita y variada gastronomía de la provincia. Además, el desarrollo económico de la provincia genera la visita de empresarios extranjeros que ven en esta zona una fuente de inversión segura. En este contexto, adicional a la preparación en primeros auxilios, los evaluadores de emergencias que laboran en el ECU 911 deben estar preparados para atender las necesidades de los visitantes extranjeros que no hablan español, y que podrían requerir los servicios de emergencia por diversas circunstancias. Este proyecto tiene como objetivo principal capacitar a los evaluadores de emergencia del ECU 911 en las destrezas comunicativas básicas del idioma inglés, utilizando para ello una metodología no formal de aprendizaje a través del Club de Inglés del proyecto de vinculación con la comunidad de la Carrera de Pedagogía de los Idiomas 
Nacionales y Extranjeros de la Facultad de Filosofía Letras y Ciencias de la Educación de la Universidad Técnica de Manabí. Esta investigación es de tipo cuasi-experimental pre test y post test de un solo grupo, obteniendo como resultado un mejoramiento en el nivel de comunicación en el idioma inglés de los beneficiarios.

Palabras clave: Club de inglés, estrategias activas y participativas, educación no formal, destrezas comunicativas del idioma inglés,

\section{ABSTRACT}

Manabí is becoming a tourist province, which is visited by thousands of people from around the world during all the year, this is because its tourist attractions are shown to the world through travel agencies and the social networks. They invite foreigners not only to visit the amazing beaches, rivers, speed bumps, parks, and museums in this city, but also to taste a variety and delicious gastronomy found in the province. Besides, the economy development of the province makes that many foreign investors visit the city, looking for creating new sources of inversion and business. In this context, besides the preparation in first aids, the emergency evaluators who work in the ECU-911 from Portoviejo should be ready to fulfill the foreigners' necessities who cannot communicate in Spanish, and who could need emergency services in any circumstances. This project has as main objective to train the emergency evaluators from ECU-911 in the use of basic communicative skills of the English language by using a nonformal learning methodology based on an English club, which is part of the community project of the Pedagogy of National and Foreign Languages Major from the Faculty of Letras y Ciencias de la Educación from the Universidad Técnica de Manabí. This investigation belongs to the quasi-experimental type, which uses a pre and post-test with one group, and the results obtained show a meaningful improvement in the communication level of the emergency evaluators that belong to ECU-911.

Keywords: English club, active and participative strategies, no formal education, English communicative skills.

\section{EL CLUB DE INGLÉS \\ DESCRIPCIÓN DEL PROBLEMA}

El aprendizaje de una lengua extranjera tiene como propósito la comunicación efectiva y eficiente, para lograr esta finalidad, los procesos de enseñanza-aprendizaje deben ser comunicativos. No se puede aprender a nadar sin sumergirse en el agua, no se puede aprender a leer sin estar en contacto con los libros, y no se puede aprender a hablar sin interactuar con otras personas; por lo tanto, las clases de inglés deben ser totalmente comunicativas. El docente debe buscar las estrategias más idóneas, de forma que los estudiantes tengan la oportunidad de expresarse.

Sin embargo, existen datos preocupantes con respecto al nivel de inglés de los docentes del Ecuador. Según el Observatorio de la Educación de la Universidad Nacional de Educación (UNAE), citado en Primicias (2019), revela que el 
$71 \%$ de los profesores de inglés del país tiene un nivel entre básico e intermedio. De acuerdo con este mismo medio de comunicación, el examen EF English Proficiency Index 2019 reveló que el país cuenta con "muy bajo nivel de competencia de inglés" debido a la falta de capacidad de sus docentes y al poco conocimiento del idioma que tienen sus estudiantes. Estos datos se evidencian en los resultados de las pruebas a los estudiantes que, según el mismo estudio, obtuvieron una calificación de 44,47/100 en el idioma inglés en la provincia de Manabí.

Los resultados de las pruebas de inglés que ha realizado el Ministerio de Educación del Ecuador han evidenciado falencias en el manejo del idioma a nivel de educación general básica y bachillerato. Con este antecedente, no es de extrañar que los evaluadores de emergencia del ECU 911 tengan las mismas dificultades, es decir, que su capacidad de comunicarse en un nivel básico es deficiente, lo cual limita su desempeño en la atención a emergencias cuando quienes la solicitan son extranjeros que no hablan español, siendo la mayoría de ellos del grupo de atención prioritaria, pues son jubilados radicados en esta provincia.

Con base en lo expuesto, el presente trabajo se plantea como objetivo determinar la relación entre los procesos educativos no formales del Club de Inglés y el desarrollo de las destrezas comunicativas de los evaluadores del ECU 911 de Portoviejo, utilizando una metodología cuasi-experimental con estrategias activas y participativas durante el proceso.

\section{SÍNTESIS Y CONCLUSIONES PRÁCTICAS}

- La prueba diagnóstica o pre test que se aplicó reveló que los participantes tenían un nivel de desempeño muy bajo en las destrezas comunicativas del idioma de acuerdo al Marco Común Europeo de Referencias para las lenguas: aprendizaje, enseñanza y evaluación.

- Al finalizar las 13 sesiones del Club de Inglés se aplicó un post test a los 21 participantes, el cual estaba basado en los estándares establecidos por el MCER para el nivel A1.

- Los resultados de esta prueba determinaron que los 21 participantes alcanzaron el nivel de usuario básico en las cuatro destrezas comunicativas del idioma inglés.

- El enfoque no formal del club de inglés, alejado de las estrictas planificaciones escolares, y más bien concebido a través de estrategias activas y participativas, permite construir un ambiente amigable, confortable y cálido, donde los asistentes se sienten seguros y animados a participar de todas las actividades, apenas sin darse cuenta de que estas son diseñadas para crear aprendizaje.

- Con los resultados obtenidos se demuestra que el uso de metodologías no formales a través del Club de Inglés, con estrategias activas y participativas, permitió el desarrollo de las destrezas comunicativas de los evaluadores del ECU 911 de Portoviejo. 


\section{INTRODUCCIÓN}

Esta investigación fue realizada en el marco del proyecto de vinculación con la sociedad denominado Fortalecimiento en Idiomas Extranjeros a los Evaluadores de Operaciones del Centro Zonal ECU 911 Portoviejo, desarrollado por la Carrera de Pedagogía de los Idiomas Nacionales y Extranjeros de la Facultad de Filosofía, Letras y Ciencias de la Educación de la Universidad Técnica de Manabí. La pregunta de investigación planteada fue: ¿̇Eiste relación entre los procesos educativos no formales del Club de Inglés y el desarrollo de las destrezas comunicativas de los evaluadores del ECU 911 de Portoviejo?

Para dar respuesta a esta pregunta se estableció como objetivo general determinar la relación entre los procesos educativos no formales del club de inglés y el desarrollo de las destrezas comunicativas de los evaluadores del ECU 911 de Portoviejo; y como objetivos específicos:

- Diagnosticar el nivel de inglés de los evaluadores de emergencias del ECU 911 del cantón Portoviejo.

- Aplicar estrategias que permitan desarrollar las destrezas comunicativas del idioma inglés a los evaluadores de emergencias del ECU 911.

- Evaluar el impacto de las sesiones de los procesos educativos no formales del Club de Inglés en los evaluadores de emergencias del ECU 911.

\section{Aprendizaje en contextos no formales:}

El aprendizaje en contextos no formales no es una modalidad nueva de estudios, de hecho, ya en el año 1999, Pastor Homs propone una definición bastante amplia, que encierra las características principales de este tipo de estudios, para él la educación no formal.

Comprende todo proceso educativo diferenciado de otros procesos, organizado, sistemático, planificado específicamente en función de unos objetivos educativos determinados, llevado a cabo por grupos, personas o entidades identificables y reconocidos, que no forme parte integrante del sistema educativo legalmente establecido y que, aunque esté relacionado con él, no proporcione directamente ninguno de sus grados y titulaciones (Homs, 1999, p. 184).

Ahora bien, los radicales cambios que ha sufrido la pedagogía en los últimos años ha vuelto esta modalidad de estudios más popular, particularmente en el campo de la educación para adultos.

De acuerdo a Zagar y Pelava (2014), la educación no formal tiene dos objetivos principales: el desarrollo profesional y el desarrollo de la personalidad del individuo; estos dos objetivos no pueden separarse, ya que mediante el desarrollo profesional, en el campo que fuese, tiene un eco importante en el desarrollo de la personalidad de los individuos, es más, puede afirmarse que los cambios que sufre el individuo en relación a su personalidad dependen de su avance o cambios en el aspecto profesional. Esta sinergia entre el campo profesional y la personalidad, que se desarrolla en los ámbitos educativos no formales, favorecen el 
desarrollo del pensamiento crítico e independiente, las destrezas sociales, la creatividad y la identidad, (Hong, 2020).

Un aspecto muy importante del éxito en los contextos no formales es la motivación, de hecho, en un estudio realizado en Finlandia se estableció que los principales resultados de aprendizaje fueron logrados, directa e indirectamente por los factores motivacionales, Hannu \& Thuneberg, (2019). La motivación principal para aprender una segunda lengua es lograr la comunicación con angloparlantes, lo cual explica la razón de que miles de usuarios estén tan interesados en las destrezas comunicativas de los idiomas, más que en su sintaxis o gramática (Zhang, 2009).

\section{Los clubes de inglés}

En el caso de inglés como lengua extranjera, la educación no formal en el marco de los clubes de inglés ha tenido presencia desde hace varios años, y se han obtenido resultados muy satisfactorios, pues los beneficiarios, al desarrollarse los procesos de aprendizaje en un ambiente no formal, se sienten seguros y confiados, lo que facilita la consecución de los objetivos, mientras que los universitarios que dirigen los clubes "adquieren una mayor responsabilidad con los aprendizajes propios, y tienen ante sí el desafío de articular los saberes aprendidos en el aula con los que demanda la praxis" (Bravo \& Castellados, 2017 p. 5).

De acuerdo con Marinova, Marshall and Snow (2020) un club de inglés es una organización de personas que comparten un propósito o un interés común, quienes se reúnen regularmente para tomar parte y compartir actividades en relación a ese propósito o interés compartido. Por su parte, Elsadig \& Hamdan (2019) afirman que el club de inglés es un espacio que permite a los usuarios utilizar el idioma en un contexto casual y no formal; esto implica que los participantes del club tienen la oportunidad de expresar sus ideas utilizando al idioma inglés en un marco ameno y amigable, en cada sesión son motivados a utilizar el idioma, a través de estrategias divertidas y dinámicas que además, fomentan el trabajo de equipo.

El Club de Inglés genera importantes ventajas para quienes están interesados en aprender esta lengua; estimula a los participantes a practicar un inglés auténtico que se utiliza en contextos de la cotidianidad, promueve la confianza entre los participantes, y mejora las competencias comunicativas mediante la creación de una atmósfera socialmente amigable entre los participantes.

Marinova, Marshall and Snow (2020) proponen algunos principios para garantizar el éxito del club de inglés:

- Las sesiones del club de inglés deben estar reguladas de acuerdo a un horario establecido.

- Las sesiones deben desarrollarse en un ambiente relajado para que los participantes practiquen su inglés.

- Tanto los integrantes como los instructores deben utilizar el idioma inglés para comunicarse.

- Todas las sesiones del club deben ser centradas en los participantes.

(1)




\section{El club de inglés y la universidad}

La universidad ecuatoriana, regida por el Consejo de Educación Superior, tiene entre sus objetivos del régimen académico "Articular la formación académica y profesional, la investigación científica, tecnológica y social, y la vinculación con la colectividad, en un marco de calidad, innovación y pertinencia." e "Impulsar el conocimiento de carácter multi, inter y trans disciplinario en la formación de grado y posgrado, la investigación y la vinculación con la colectividad". Reglamento de Régimen Académico Consejo Educación Superior, (2017 p. 3), lo cual implica que todas las universidades del Ecuador deben relacionarse con la comunidad a través de proyectos de vinculación, con la finalidad de contribuir a la solución de sus problemas.

En este contexto, la Universidad Técnica de Manabí se propone en su misión "Formar académicos, científicos y profesionales responsables, humanistas, éticos y solidarios, comprometidos con los objetivos del desarrollo nacional, que contribuyan a la solución de los problemas del país como universidad de docencia con investigación, capaces de generar y aplicar nuevos conocimientos, fomentando la promoción y difusión de los saberes y las culturas, previstos en la Constitución de la República del Ecuador" (Estatuto Orgánico de la Universidad Técnica de Manabí, 2013, p. 5), e indica entre sus objetivos la necesidad de "Realizar actividades de vinculación con la sociedad, orientadas a desarrollar su trabajo académico en todos los sectores" (Estatuto Orgánico de la Universidad Técnica de Manabí, 2013 p. 6). Todo ello impulsa a las diferentes carreras universitarias a intervenir en los grupos estratégicos de la sociedad estableciendo una sinergia entre sus necesidades y las aportaciones que la universidad puede hacer a través de los alumnos que están realizando sus estudios de educación superior.

Entre las múltiples intervenciones en que participa la universidad está la de formación de clubes de inglés con personas interesadas en aprender este idioma quienes, por opción, han participado sin rango de edad, género o clase social. La Universidad Técnica de Manabí tiene experiencia en el desarrollo de clubes de inglés, tal como lo sustentan las conclusiones de Mendoza, K. (2018) al argumentar la necesidad de sistematizar la experiencia formativa de los clubes de inglés a través de manuales que contengan nuevas estrategias que favorezcan el aprendizaje de este idioma.

\section{Estrategias activas y participativas.}

Estrategias metodológicas activas y participativas son aquellas en donde los estudiantes actúan como actores principales en el proceso de enseñanza aprendizaje de inglés como lengua extranjera. Como el director dirige con su batuta los acordes de cada instrumento en una orquesta sinfónica conformada por cuarenta o cincuenta personas, así el maestro dirige con instrucciones claras y precisas las actividades de cada uno de los alumnos que participan en su clase. No es el profesor el que debe realizar las actividades o ensayar los ejercicios, sino que es aquel que monitorea la actividad de los alumnos, el que guía el proceso de forma que todos participen, evaluando el desempeño de cada uno de los participantes y brindando el apoyo efectivo en caso de que sea necesario (Cedeño, 2013). 
Las estrategias metodológicas activas y participativas responden a una planificación efectiva y consciente, donde se tome en cuenta los diversos aspectos que comprende el manejo de aula de clases, tales como el número de estudiantes, su edad, la dimensión del aula de clases, los equipos tecnológicos que esta tiene, el tipo de mobiliario, y por supuesto los recursos didácticos. El éxito de toda clase de inglés es una correcta planificación, y cuando se trata del tipo de actividades que nos ocupa, los planes de lección juegan un papel aún más importante. Para ejemplificar, imagine haber planificado una actividad en la cual los estudiantes deben escuchar una canción para obtener las instrucciones que deben seguir, por lo tanto, se requiere el uso de una computadora y parlantes. Si el docente cuenta con esos equipos en el aula de clases, no tendrá ningún problema; si no los tiene, obedeciendo a su planificación tendrá que llevar al aula una grabadora u otro equipo de audio, pero si no lo ha considerado en su planificación, la clase se verá boicoteada por la falta de estos equipos que no fue prevista por el profesor, perdiéndose la efectividad de las estrategias planificadas (Cedeño, 2013).

Este tipo de estrategias tiene muchas utilidades a la hora de desarrollar procesos de interaprendizaje efectivos, "aunque la principal, tal vez sea lograr la participación del alumno en sus propios procesos de aprendizaje y adquisición de conocimiento" al decir de López (2007 p. 108).

Para Barroso, Barroso y Parra (2013, p. 11), las estrategias activas y participativas ayudan a alcanzar objetivos genéricos de educación tales como: "la motivación o el interés por el aprendizaje, la comprensión de lo que se estudia, la participación activa en el proceso de enseñanza aprendizaje, la aplicación de lo visto en situaciones de la vida real". Por otra parte, al decir de estos autores, aunque el acto de aprender es algo que se debe tomar en serio, no es indispensable estar serios para hacerlo, ya que se puede aprender jugando, divirtiéndose, aprovechando la tendencia innata que todo organismo tiene para disfrutar aquello que les gusta.

\section{MATERIALES Y MÉTODOS}

Esta investigación se llevó a cabo con veintiún evaluadores de emergencia del Servicio Integrado de Seguridad ECU 911 -Portoviejo, el cual está ubicado en la Parroquia Andrés de Vera del cantón Portoviejo, provincia de Manabí en la República del Ecuador.

Para realizar el presente estudio, se utilizaron materiales bibliográficos con el objetivo de sustentar científicamente las variables y elaborar las planificaciones de las sesiones del Club de Inglés.

\section{Método}

El método utilizado para esta investigación fue el correlacional, el cual según Sampieri "tiene como finalidad conocer la relación o grado de asociación que exista entre dos o más conceptos, categorías o variables en una muestra o contexto en particular" (2014, p. 93), y este proyecto pretende relacionar el método no formal de estudios del Club de Inglés y el desarrollo de las destrezas comunicativas del idioma.

.

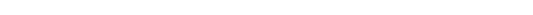




\section{Metodología}

Esta investigación es de tipo cuasi-experimental pre test y post test de un solo grupo (Ñautas, Mejía, Noboa y Villagómez, 2013). Se aplicó una prueba diagnóstica antes de iniciar las sesiones del club y otra al término de las mismas con la intención de analizar el avance de los participantes en cuanto a las destrezas comunicativas del idioma inglés.

El pre test que se aplicó al inicio de las sesiones consistió en una prueba de inglés que contenía gramática básica: verbo to be y tiempo presente simple. También contenía vocabulario básico relacionado con el ambiente y descripciones personales y de los lugares comunes.

El post test en su parte inicial contenía los mismos elementos del pre test y se agregaron además dos actividades, una de producción oral y otra de producción escrita, en las cuales los participantes pudieron expresarse utilizando un nivel muy básico del idioma.

Para desarrollar este estudio se escogieron 8 alumnos del quinto nivel de la Carrera de Pedagogía de los Idiomas Nacionales y Extranjeros, quienes, de acuerdo al Marco Común Europeo, ya son usuarios independientes del idioma y ya habían sido expuestos a asignaturas que convergen el uso del inglés y la didáctica para su aprendizaje; estos alumnos se convirtieron en tutores en las sesiones del club.

Las sesiones del Club de Inglés iniciaron el 5 de noviembre de 2019 con 21 participantes organizados en 4 grupos, de acuerdo a la disponibilidad de sus horarios de labores. Las sesiones duraban 4 horas, de 14:00 a 18:00, en las que se desarrollaron temas comunicativos mediante el uso de estrategias activas y participativas que permitieron la interacción de los beneficiarios y favorecieron su comunicación.

En el siguiente cuadro se detallan los objetivos de cada sesión:

\section{Tabla 1}

Resultados de la prueba de entrada o pre test aplicada a los evaluadores de emergencias del ECU 911 de Portoviejo

\begin{tabular}{|c|c|}
\hline SEMANA & OBJETIVOS \\
\hline 1 & $\begin{array}{l}\text { Presentarse a sí mismo y a sus compañeros } \\
\text { dando información personal. }\end{array}$ \\
\hline 2 & $\begin{array}{l}\text { Describir a los miembros de la familia } \\
\text { y sus ocupaciones. }\end{array}$ \\
\hline 3 & $\begin{array}{l}\text { Expresar gustos, disgustos y preferencias en } \\
\text { relación a comida y bebida. }\end{array}$ \\
\hline 4 & $\begin{array}{l}\text { Describir a una persona en relación a su } \\
\text { apariencia y a su personalidad. }\end{array}$ \\
\hline 5 & $\begin{array}{l}\text { Describir elementos que hay o no en las diferentes } \\
\text { dependencias de una casa y otros lugares. }\end{array}$ \\
\hline 6 & $\begin{array}{l}\text { Exponer su rutina diaria expresando lo que hace } \\
\text { regularmente y los tiempos en que lo ejecuta. }\end{array}$ \\
\hline 7 & $\begin{array}{l}\text { Describir celebraciones y las actividades comunes } \\
\text { realizadas en ellas. }\end{array}$ \\
\hline 8 & $\begin{array}{l}\text { Leer descripciones cortas sobre personas, rutinas y celebraciones } \\
\text { para obtener e inferir información relevante sobre lo leído. }\end{array}$ \\
\hline 9 & $\begin{array}{l}\text { Describir hechos y acciones cuando estos } \\
\text { ocurran. Describir fotografías e imágenes. }\end{array}$ \\
\hline 10 & $\begin{array}{l}\text { Describir actividades, } \\
\text { vestuarios y accesorios. }\end{array}$ \\
\hline 11 & $\begin{array}{l}\text { Desarrollar diálogos comunes } \\
\text { en una terminal aérea }\end{array}$ \\
\hline 12 & $\begin{array}{l}\text { Describir accidentes y enfermedades comunes. } \\
\text { Dar sugerencias }\end{array}$ \\
\hline 13 & $\begin{array}{l}\text { Desarrollar diálogos comunes en una } \\
\text { llamada de emergencia. }\end{array}$ \\
\hline
\end{tabular}

Nota. Objetivos de 13 sesiones realizadas. 


\section{RESULTADOS}

Tabla 2

Notas del examen de entrada sobre 100

\begin{tabular}{c|ccc}
\multicolumn{2}{c}{ No. } & Frecuencia & $\%$ \\
\hline \multirow{4}{*}{$\begin{array}{c}\text { Notas del } \\
\text { examen de } \\
\text { entrada }\end{array}$} & $91-100$ & 0 & 0 \\
\cline { 2 - 4 } sobre 100 & $81-90$ & 0 & 0 \\
\cline { 2 - 4 } & $71-80$ & 1 & 4,76 \\
\cline { 2 - 4 } & $61-70$ & 2 & 9,52 \\
\cline { 2 - 4 } & $41-60$ & 3 & 14,30 \\
\cline { 2 - 4 } & $21-40$ & 0 & 9,52 \\
\cline { 2 - 4 } & $11-20$ & 11 & 0 \\
\cline { 2 - 4 } & $1-10$ & 2 & 92,38 \\
\cline { 2 - 4 } & & 21 & $100 \%$ \\
\hline
\end{tabular}

Nota. Resultados de la aplicación de pruebas de diagnóstico a los interesados en participar.

Previo al inicio de las sesiones del Club de Inglés se aplicaron pruebas diagnósticas a los interesados en participar. Esta prueba contenía frases muy básicas y cotidianas, discurso lento y articulado cuidadosamente con largas pausas, textos muy breves y simples, nombres familiares y palabras de acuerdo al nivel A1 del Marco Común Europeo de Referencias para la Enseñanza y Aprendizaje de Lenguas. Veintiún operadores de emergencia se presentaron a la prueba de diagnóstico, la cual estaba valorada sobre cien puntos, y ninguno alcanzó el nivel de comprensión básica de acuerdo con el MCER.
Se logró diseñar las trece sesiones del Club de Inglés atendiendo las necesidades de los beneficiarios de manera que ellos pudieran desarrollar sus destrezas comunicativas, mismas que se realizaron con base en la planificación microcurricular vigente en el país. La satisfacción y efectividad de las sesiones del Club de Inglés fueron validadas por los beneficiarios, concluyendo que el curso cubría las expectativas promoviendo la participación, que la metodología aplicada por los facilitadores estuvo acorde a las exigencias del curso, que los facilitadores estaban completamente preparados para liderar las sesiones, que los materiales didácticos, y que los recursos utilizados fueron adecuados y originales, lo cual repercutió en el incremento de su nivel de inglés.

\section{Tabla 3}

Resultados de la prueba de salida o post test aplicada a los evaluadores de emergencias del ECU 911 de Portoviejo

\begin{tabular}{c|ccc}
\multicolumn{2}{c}{ No. } & Frecuencia & $\%$ \\
\cline { 2 - 4 } Notas del & $91-100$ & 4 & 19,04 \\
\cline { 2 - 4 } examen de & $81-90$ & 5 & 23,81 \\
\cline { 2 - 4 } $\begin{array}{c}\text { entrada } \\
\text { sobre 100 }\end{array}$ & $71-80$ & 9 & 42,86 \\
\cline { 2 - 4 } & $61-70$ & 3 & 14,29 \\
\cline { 2 - 4 } & & 21 & $100 \%$ \\
\hline
\end{tabular}

Nota. Resultados de satisfacción y efectividad de las sesiones del Club de Inglés. 


\section{Tabla 4}

Resultados comparativos del pre test y post test

\begin{tabular}{|c|c|c|c|c|}
\hline & \multicolumn{3}{|c|}{ RESULTADOS } & \\
\hline & PRE TEST & & POST TEST & \\
\hline & 54 & & 100 & \\
\hline & 28 & & 98 & \\
\hline & 74 & & 98 & \\
\hline & 40 & & 94 & \\
\hline & 58 & & 90 & \\
\hline & 28 & & 89 & \\
\hline & 68 & & 85 & \\
\hline & 60 & & 82 & \\
\hline & 44 & & 81 & \\
\hline & 24 & & 78 & \\
\hline & 52 & & 78 & \\
\hline & 28 & & 77 & \\
\hline & 22 & & 74 & \\
\hline & 24 & & 74 & \\
\hline & 24 & & 73 & \\
\hline & 48 & & 72 & \\
\hline & 18 & & 71 & \\
\hline & 24 & & 71 & \\
\hline & 30 & & 63 & \\
\hline & 20 & & 68 & \\
\hline & 26 & & 51 & \\
\hline & PRE TEST & & POST TEST & \\
\hline & MEDIA $=37,18$ & & MEDIA $=79,38$ & \\
\hline & MEDIANA $=2 \varepsilon$ & & MEDIANA $=78$ & \\
\hline & $M O D A=24$ & & MODA $=98$ & \\
\hline GRUPOS & $\begin{array}{l}\text { COMPOSICIÓN } \\
\text { DE LOS GRUPOS }\end{array}$ & $\begin{array}{l}\text { MEDIA } \\
\text { PRE TEST }\end{array}$ & $\begin{array}{l}\text { VARIABLE } \\
\text { INDEPENDIENTE }\end{array}$ & $\begin{array}{l}\text { MEDIA } \\
\text { POST TEST }\end{array}$ \\
\hline 1 & 21 & 37,18 & $\begin{array}{c}\text { ESTRATEGIAS ACTIVAS } \\
\text { Y PARTICIPATIVAS }\end{array}$ & 79,38 \\
\hline
\end{tabular}

Nota. Resultados de test antes y después de ejecutar las sesiones. Barretos, (2013, p. 99)

La diferencia de las medias entre el pre test y post test es de 42,2 puntos; lo cual devela que las estrategias activas y participativas utilizadas para la enseñanza del idioma a través de los clubes de inglés a los evaluadores del ECU 911, lograron resultados significativamente diferentes en ambas evaluaciones demostrando la efectividad de las mismas en el entorno que se las aplicó.

\section{DISCUSIÓN}

Al finalizar las sesiones del Club de Inglés con los evaluadores del ECU 911 de Portoviejo, con la aplicación de la prueba de salida, se visualiza que cuatro beneficiarios (lo que corresponde al 19,04\%) obtuvieron una calificación de más de 90 puntos en la mencionada prueba; cinco evaluadores del ECU 911 (es decir, el 23,81 \%) tuvieron una calificación de entre ochenta a noventa puntos sobre cien, y nueve operadores (el 42,86 \%) consiguieron una puntuación de entre 70 a 80 puntos. Estos datos implican que dieciocho de los veintiún participantes, es decir, el 81,71\% obtuvieron puntajes de más de setenta puntos, lo que expone los beneficios de las sesiones del Club de Inglés, cuyo objetivo principal era determinar la relación entre los procesos educativos no formales del club y el desarrollo de las destrezas comunicativas de los evaluadores del ECU 911 de Portoviejo, quienes, como lo demuestra la prueba, alcanzaron los objetivos de comunicación elemental de acuerdo con el Marco Común Europeo, los cuales proponen que los usuarios puedan presentarse de forma sencilla y usar saludos básicos, es decir, de donde son ellos y otras personas y dar una descripción de su ciudad, hablar de manera simple sobre la familia, describir rasgos de su aspecto y de su personalidad, describir la ropa en un nivel básico, expresar preguntas al 
dependiente en una tienda, hablar sobre sus hobbies, intereses, gustos de su familia y amigos, y entender temas relacionados con su entorno.

Todos estos aspectos fueron verificados a través de la prueba de post test que contenía preguntas de expresión escrita y oral sobre los temas expuestos y que propone el Marco Común Europeo de Referencias como un hablante de nivel Al del idioma inglés.

Estos resultados demuestran también la efectividad del uso de estrategias activas y participativas a través del Club de Inglés, ya que se determinó que el progreso de los evaluadores del ECU 911 es significativo, pues fueron capaces de obtener información y de describir situaciones y hechos de la cotidianidad utilizando este idioma, lo cual está acorde con los objetivos que el Marco Común Europeo propone para el nivel Al de usuarios del inglés.

\section{CONCLUSIONES}

Con los resultados evidenciados en las pruebas, luego de las sesiones del Club de Inglés, se ha llegado a las siguientes conclusiones:

Existe una evidente relación entre los procesos educativos no formales del Club de Inglés y el desarrollo de las destrezas comunicativas de los evaluadores del ECU 911 de Portoviejo, considerando los resultados de la evaluación de salida aplicada al personal del Servicio Integrado de Seguridad ECU 91 1, inmersos en este proyecto.
El ambiente amigable que genera el Club de Inglés permite que los participantes adquieran destrezas comunicativas del idioma para expresarse, es decir, que no solo memorizan vocabulario y gramática, sino que son capaces de describir hechos concretos de la vida cotidiana, así como expresar pensamientos simples y abstractos, tal como lo indica el Marco Común Europeo para los aprendices de nivel Al de inglés como idioma extranjero.

Los aprendientes expuestos a entornos de educación no formal en los clubes de inglés, y el uso de estrategias activas y participativas, contribuyen a mejorar las destrezas comunicativas en los evaluadores de manera significativa.

Las sesiones del Club de Inglés correspondieron a cincuenta y dos horas en trece periodos, los cuales fueron planificados de acuerdo con los objetivos que describe el MCER, sin embargo, y a pesar de haberse logrado los objetivos propuestos en este estudio, se sugiere un incremento en el número de sesiones, de manera que haya más tiempo para la práctica de las destrezas comunicativas del idioma y el afianzamiento de sus funciones.

Los resultados obtenidos en este estudio recomiendan que se planifiquen nuevos clubes de inglés que incorporen todos los niveles establecidos por el MCER, de manera que los evaluadores del ECU 911 puedan participar de ellos, y en el proceso lograr el nivel de usuarios independientes de este idioma, con lo cual podrían brindar mejor servicio a los beneficiarios que requieren los servicios de esta institución. 
El impacto del Club de Inglés contribuye a que los evaluadores de emergencias del ECU 911 brinden una atención eficiente y oportuna, acorde a la política de calidad que tienen, enfocada en la mejora continua para la búsqueda de la satisfacción de los usuarios en el territorio ecuatoriano, por lo tanto, es recomendable que los clubes de inglés se conviertan en una actividad permanente de capacitación.

\section{AGRADECIMIENTOS}

"Trabajar en equipo divide el trabajo y multiplica los resultados"

Anónimo

La complejidad laboral se simplifica cuando los miembros de un equipo trabajan en armonía y buscan el beneficio común. Esta premisa, garantiza el cumplimiento de objetivos planteados, garantizando resultados de calidad que, en muchos casos, superan los estándares establecidos.

Este trabajo investigativo reúne estas condiciones y por ello ha logrado resultados significativos que benefician a participantes y beneficiarios del Proyecto de Vinculación con la Sociedad que se realizó a través del Club de Idiomas de la Universidad Técnica de Manabí en el ECU 911 de Portoviejo.

Quienes hemos realizado este artículo científico nos llenamos de complacencia por los resultados obtenidos y queremos agradecer profundamente a las personas que dieron su mejor esfuerzo en la realización de este proyecto.
- Carrera de Pedagogía de los Idiomas Nacionales y Extranjeros de la Universidad Técnica de Manabí, por ser la gestora de este proyecto de Vinculación con la Sociedad.

- Al Servicio Integrado de Seguridad ECU 911, por permitir la ejecución del proyecto y especialmente al Ing. Gabriel Cevallos, jefe de Operadores de esta prestigiosa institución.

- A los estudiantes participantes/docentes de la carrera PINE, que dieron su mejor esfuerzo plasmado en una enseñanza armónica y de calidad.

- A los operadores de servicio, estudiantes y beneficiarios, que demostraron compromiso hacia el aprendizaje reflejado en el extraordinario nivel de inglés alcanzado.

Reiteramos nuestro compromiso de seguir trabajando en servicio de la colectividad, y continuar siendo parte fundamental del desarrollo de nuestra comunidad, cumplir con la misión de formar personas en el manejo de los idiomas para crear una cultura de aprendizaje entre sus habitantes.

Los autores

\section{REFERENCIAS}

Barroso, A. Barroso, R. \& Parra, G. (2013). Las dinámicas grupales y el proceso de aprendizaje. Instituto Politécnico Nacional. México. http://www.repositoriodigital.ipn.mx/ handle/123456789/17489 
Cedeño, L. (2013). El uso de estrategias metodológicas activas y participativas en la enseñanza-aprendizaje del inglés como lengua extranjera y su incidencia en la adquisición de la comprensión lectora en los estudiantes del Instituto de Lenguas de la Universidad Técnica de Manabí. (Tesis doctoral inédita), Universidad Nacional Mayor de San Marcos, Lima

Common European Framework of Reference for Languages: Learning, teaching, assessment. Council o Europe. https://www.coe.int/t/ dg4/linguistic/Source/Framework_EN.pdf

EF. EPI. (2020). ef.com.ec. Recuperado el 19 de Septiembre de 2020, de https://www.ef.com.ec/epi/regions/latin-america/ecuador/

Elsadig, E. \& Hamdan, A. (2019). The Effectiveness of English Club as Free Voluntary Speaking Activity Strategy in Fostering Speaking Skill in Saudi Arabia Context. International Journal of Linguistics, Literature and Translation (IJLLT) ISSN: 2617 0299, IJLLT 2(1):230-235. https://files.eric.ed.gov/fulltext/ ED593449.pdf

Estatuto Orgánico de la Universidad Técnica de Manabí, (2013). https://issuu.com/publicacionesutm/docs/estatutoorganico

Hannu, H. \& Thuneberg, H. (abril, 2019). The Role of SelfDetermination in Informal and Formal Science Learning Contexts, Learning Environment. p21 https://eric. ed.gov/?q=no + formal + contexts\&id $=$ EJ 1208996

Hong, H. (abril de 2020). Towards a Synthesis of Formal, Non-Formal and Informal Pedagogies in Popular Music Learning. Research Studies in Music Education. v42 n 1 p56-76 Apr 2020. https:// eric.e d.gov/ ? $\mathrm{q}=$ no + formal+contexts + for+teaching + engish\&id $=$ EJ1250655
López, F. (2007). Metodología participativa en la enseñanza universitaria. Narcea S.A de ediciones. Madrid, España. books. google.co.in/books?isbn=842771498X

Marinova-Todd, S., D. Marshall, and C. Snow. 2000. Three misconceptions about age and L2 learning. TESOL Quarterly, 34 (1): 9-34. https://personal.utdallas.edu/ assmann/ hcs6367/marinova_todd_marshall_snow00.pdf

Mendoza, K. \& Castellanos, A. (2017). Una mirada crítica a la experiencia en el Club de Inglés desde la dimensión pedagógica de la extensión universitaria. Revista Cubana de Educación Superior. Vol 34. N 3. http://scielo.sld. cu/scielo.php? script=sci_arttext\&pid=S0257-4314 2017000300012

Mendoza, K. (2018). Alternativa metodológica para la práctica extensionista club de inglés como experiencia formativa de compromiso social en la Universidad Técnica de Manabí Ecuador. (Tesis doctoral inédita). Universidad de la Habana. Cuba.

Nygaard, C. Holtham, C \& Courtney, N. (2009) Improving student's learning outcomes. Copenhagen Bussiness School Press. Denmark. https://books.google.com.ec/ books? id =o5Fd5-YQe1EC\&printsec $=$ frontcover\&dq $=1 \mathrm{~m}$ proving + student\%E2\%80\%99s + learning + outcomes\&hl $=e s \& s a=X \&$ redir esc $=y \# v=$ onepage $\& q=I m p r o v i n g \% 20$ student\%E2\%80\%99s\%20learning\%20outcomes $\& f=$ false

Ñautas, H. Mejía, E. Noboa, E. \& Villagómez, A. (2013). Metodología de la investigación científica y elaboración de tesis. Ed. Universidad Mayor de San Marcos. Lima, Perú. 
Pastor Homs, M. I. (1999). Ámbitos de intervención en Educación no formal. Una propuesta taxonómica. Revista Teoría de la Educación. Ediciones Universidad de Salamanca, 1 1, 183-215

Primicias.ec. (2019). Primicias.ec. Recuperado el 19 de Septiembre de 2020, de https://www.primicias.ec/noticias/sociedad/ idioma-ingles-estudiantes-convenio-educacion-profesores/

Reglamento de Régimen Académico Consejo Educación Superior. (2017), República del Ecuador. https://www. ces.gob.ec/lotaip/2018/Enero/Anexos\%20Procu/Anlit-a 2 -Reglamento\%20de\%20R\%C3\%A9gimen $\% 20$ Acad\%C3\%A9mico.pdf

Sampieri, R. (2014). Metodología de la investigación. 6ta edición. Ed. Mc Graw Hill. México. http://observatorio.epacartagena. gov.co/wp-content/uploads/2017/08/metodologia-de-lainvestigacion-sexta-edicion.compressed.pdf

Zagar, I. \& Kelava, P. (2014). From Formal to Non Formal Education, Learning and Knowledge. Cambridge Schollars Publishing. United Kingdom. https://books. google.com.ec/ books? id =icsxBwAAQBAJ\&printsec $=$ frontcover\&dq $=$ no + formal + education\&hl $=$ es\&sa $=X \& v e d=$ OahUKEwilnqP199noAhV hUd8KHXN2ATAQ 6AEISjAD\#v= onepage $\& q=$ no\%20formal\%20education $\& f=$ false

Zhang, Y. (2009). Reading to speak. Integrating oral communicative skills. English Teaching Forum. p. 32. https://files.eric.ed.gov/ fulltext/EJ923446.pdf 


\section{BIOGRAFÍA DE LOS AUTORES}

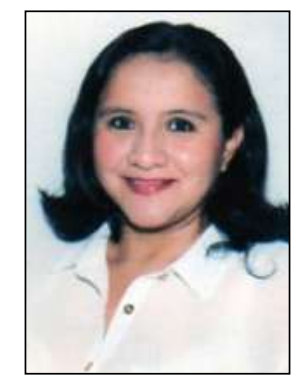

\section{LETICIA MERCEDES CEDEÑO MACÍAS}

Profesora de inglés como lengua extranjera, con veinte años de experiencia docente. Tiene certificación TKT y ICELT de la Universidad de Cambridge, también tiene una Maestría en Investigación y Docencia Educativa de la Universidad Técnica de Manabí y un Doctorado de la Universidad San Marcos en Perú.

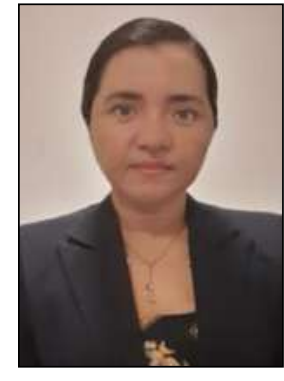

\section{ROSALYN YAHAIRA CEDEÑO ZAMBRANO}

Magíster en Docencia e Investigación Educativa por la Universidad Técnica de Manabí (UTM). Docente de la UTM, posee certificación TEFL, TESL y ICELT. Es profesora de inglés como lengua extranjera con veinte años de experiencia en todos los niveles educativos, tanto públicos como privados.

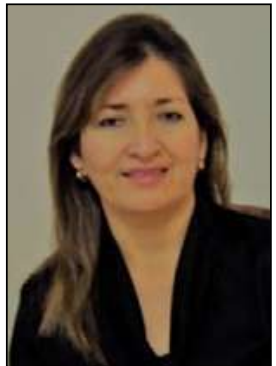

DRA. ÁNGELA FLORES VÉLEZ
Docente de la Carrera de Pedagogía de los Idiomas Nacionales y Extranjeros de la Universidad Técnica de Manabí. Profesora certificada en la enseñanza de inglés por la Universidad de Cambridge, con dieciocho años de experiencia, Máster en Docencia e Investigación y Ph. D. en Educación Superior.

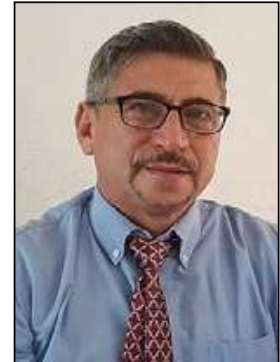

MTR. JOEL BALDOMERO LOOR PONCE

Docente agregado a tiempo completo de la Universidad Técnica de Manabí, Vicedecano de la Carrera de Pedagogía de los Idiomas Nacionales y Extranjeros - UTM, graduado de esta misma universidad en la Licenciatura en Ciencias de la Educación y Maestría en Docencia e Investigación Educativa 